\title{
Non-perturbative gauge-fixing
}

\author{
Asit K. De and Mugdha Sarkar* \\ Theory Division, Saha Institute of Nuclear Physics, HBNI, \\ 1/AF Bidhannagar, Kolkata 700064, India \\ E-mail: asitk.deesaha.ac.in \\ E-mail: mugdha.sarkaresaha.ac.in
}

For theories on lattice such as manifestly local chiral gauge theories, the longitudinal gauge degrees of freedom affect the dynamics of the physical sector in an undesirable manner. However, to control these redundant degrees of freedom, the standard Fadeev-Popov gauge-fixing procedure cannot be employed, since the Becchi-Rouet-Stora-Tyutin (BRST) symmetry of the off-shell Fadeev-Popov action with compact lattice gauge fields leads to an indeterminate $0 / 0$ form for expectation value of any gauge-invariant operator. This work describes numerical investigation of proposals that evade the above no-go situation for both Abelian and non-Abelian lattice gauge theories.

The 36th Annual International Symposium on Lattice Field Theory - LATTICE2018

22-28 July, 2018

Michigan State University, East Lansing, Michigan, USA.

\footnotetext{
${ }^{*}$ Combined contribution of both speakers
} 


\section{Introduction}

The standard lattice gauge theory as formulated by Wilson on a finite 4 dimensional hypercubic Euclidean lattice is well defined without gauge-fixing. The theory is quantised through a functional integral with a gauge-invariant measure, and gauge invariance is manifest at all stages of a nonperturbative calculation. This formalism works very well for a vector-like theory like Quantum Chromodynamics, however, meets with severe problems for manifestly local formulations of chiral gauge theories on lattice.

Lattice actions such as chiral gauge theories as mentioned above have terms that explicitly break gauge invariance. Since the functional integration integrates also along the gauge orbit, the longitudinal gauge degrees of freedom (lgdof) effectively couple with physical degrees of freedom, i.e., fermions and transverse gauge fields, in the terms that explicitly break gauge invariance. The lgdof are elements of the gauge group and are radially frozen scalar fields. In the absence of a kinetic term for the lgdof, these are random fields and the resulting strong couplings led to the failure of several proposals for lattice chiral gauge theories in the past. An obvious recipe to try out is gauge-fixing. However, non-perturbatively (i.e., with compact lattice gauge fields) it was shown that for a BRST-invariant theory the unnormalised expectation value of a gauge-invariant operator as well as the partition function is strictly zero [1]. In fact the BRST invariance makes sure that the positive and the negative sectors which arise out of multiple solutions (Gribov copies) of the gauge-fixing condition exactly cancel. If the gauge-fixing avenue is to be pursued, it is clear that something needs to be done to go around this impasse.

Our work [2, 3, 4] focusses on two non-perturbative gauge-fixing proposals, one for $U(1)$ theory and the other for $S U(N)$ gauge theories, and numerically investigate them.

\section{Compact $U(1)$ lattice gauge theory with higher derivative gauge-fixing}

\subsection{Introduction}

The proposal [5] for the $U(1)$ theory breaks BRST symmetry explicitly through a specially engineered higher derivative (HD) gauge-fixing term (on the lattice) that has a unique absolute minimum of the action and has the desired continuum limit, i.e., a covariant gauge-fixing term. By controlling the coefficient of the HD gauge-fixing term and appropriate counter-terms, it is possible to recover the BRST symmetry (and gauge symmetry in the physical sector) so that the lgdof decouple from the theory dynamically.

The $U(1)$ proposal was investigated earlier, at weak gauge couplings, both in perturbation theory and also using numerical simulation and a novel phase transition was found between a regular ordered phase (FM) and a spatially modulated ordered phase (FMD) that broke Euclidean invariance. When this phase transition was approached from FM, the lgdof were found to decouple from the theory with only free massless photons appearing in the spectrum.

In fact, $U(1)$ chiral gauge theories were investigated with this gauge-fixing method within the Smit-Swift model using Wilson fermions and a so-called waveguide model using domain-wall fermions. Both proposals famously failed previously without gauge-fixing. However, with the HD gauge-fixing, in the reduced model limit (i.e., for zero gauge coupling), these theories were able to produce chiral fermions with the scalars (lgdof) decoupled [6, 7]. 
It may be noted that the standard compact $U(1)$ lattice gauge theory has been known to have phase transition at gauge coupling $g \sim 1$. The weak coupling phase, known as the Coulomb phase, is the usual phase with free massless photons appearing in the spectrum. The strong coupling phase is confining, giving rise to non-perturbative properties like formation of gauge-balls etc. However, there exists no quantum continuum limit as the phase transition is found to be weakly first order.

Apart from the interests for a non-perturbative definition of a $U(1)$ chiral gauge theory, and also a possible alternative formulation of the standard lattice theory, the investigations at strong gauge coupling is thus also an interesting probe for any non-trivial properties of $U(1)$ gauge theory at short distances. Recently, we have investigated the HD gauge-fixing proposal at strong gauge couplings $[2,3]$.

At strong gauge couplings in the $U(1)$ case, we encountered problems with local algorithms like the Multihit Metropolis (MM) used entirely for the numerical investigations at weak gauge couplings. There were problems at larger values of the coefficient of the HD gauge-fixing term even at weak gauge couplings. We found that a Hybrid Monte Carlo (HMC), a global algorithm, performed more reliably with the HD action at stronger gauge couplings and larger coefficient of the HD term. Accurate quantitative analysis was only possible after we switched to the HMC algorithm.

\subsection{The HD gauge-fixing action and results at weak gauge coupling}

The Euclidean action, containing only physical fields, on a 4-dimensional hypercubic lattice is given by:

$$
S=S_{\mathrm{W}}+S_{\mathrm{GS}}+S_{\mathrm{ct}} .
$$

The first term in (2.1), $S_{\mathrm{W}}$, is the gauge-invariant standard Wilson term containing a summation over all gauge plaquettes $P_{x \mu \nu}$,

$$
S_{\mathrm{W}}=\frac{1}{g^{2}} \sum_{x, \mu<v}\left(1-\operatorname{Re} P_{x \mu \nu}\right),
$$

the plaquette being the smallest Wilson loop around an elementary square at a lattice point $x$ on the $(\mu, v)$ plane.

The second term in (2.1), $S_{\mathrm{GS}}$, is the Golterman-Shamir HD gauge-fixing term [5] and is given by

$$
S_{\mathrm{GS}}=\tilde{\kappa}\left(\sum_{x y z} \square_{x y}(U) \square_{y z}(U)-\sum_{x} B_{x}^{2}\right),
$$

where the gauge-covariant Laplacian $\square_{x y}(U)$ is given by,

$$
\square_{x y}(U)=\sum_{\mu}\left(\delta_{y, x+\mu} U_{x \mu}+\delta_{y, x-\mu} U_{x-\mu, \mu}^{\dagger}-2 \delta_{x y}\right),
$$

and,

$$
B_{x}=\sum_{\mu}\left(\mathscr{A}_{x-\mu, \mu}+\mathscr{A}_{x \mu}\right)^{2} / 4, \text { with } \mathscr{A}_{x \mu}=\operatorname{Im} U_{x \mu} .
$$


The action (2.1) with the HD gauge-fixing term has a unique absolute minimum at $U_{x \mu}=1$ [5] . In the naive continuum limit, the HD gauge-fixing term gives the usual covariant gauge fixing term

$$
\tilde{\kappa} g^{2} \int d^{4} x\left(\partial_{\mu} A_{\mu}\right)^{2}=(1 / 2 \xi) \int d^{4} x\left(\partial_{\mu} A_{\mu}\right)^{2},
$$

where $\xi$ is defined as

$$
\xi=1 /\left(2 \tilde{\kappa} g^{2}\right)
$$

As a result, a weak coupling perturbation theory (WCPT) of the gauge fixed theory with $\xi \sim 1$ is defined around $g=0$ and large $\tilde{\kappa} \rightarrow \infty$.

The action explicitly breaks BRST symmetry (no ghost fields present, and the HD gaugefixing term is not an exact square of the gauge-fixing function) and thus the theory evades the nogo theorem. Obviously for physical fields, gauge symmetry is broken. To recover the symmetry, remembering that HD gauge-fixing is expected to lead to a renormalisable gauge, counter-terms can be constructed using power counting. We have considered only a dimension- 2 gauge mass counter-term

$$
S_{\mathrm{ct}}=-\kappa \sum_{x \mu}\left(U_{x \mu}+U_{x \mu}^{\dagger}\right) .
$$

There are five other marginal counter-terms possible. However, they all can be treated perturbatively [8].

Under a gauge transformation $U_{x \mu} \rightarrow \mathrm{g}_{x} U_{x \mu} \mathrm{g}_{x+\mu}^{\dagger}$, the gauge non-invariant terms, collectively called $S_{\mathrm{NI}}\left[U_{x \mu}\right]\left(=S_{\mathrm{ct}}\left[U_{x \mu}\right]+S_{\mathrm{GS}}\left[U_{x \mu}\right]\right)$, pick up the lgdof and the theory becomes a scalar-gauge system. The action obtained after the gauge transformation (the so-called Higgs picture) now includes $S_{\mathrm{NI}}\left[\phi_{x}^{\dagger} U_{x \mu} \phi_{x+\mu}\right]$ with the lgdof $\phi_{x}=\mathrm{g}_{x}^{\dagger}$ radially frozen scalar fields.

The mass counter-term (2.8) takes the following form in the Higgs picture:

$$
S_{\mathrm{ct}}^{\phi}=-\kappa \sum_{x \mu}\left(\phi_{x}^{\dagger} U_{x \mu} \phi_{x+\mu}+\phi_{x+\mu}^{\dagger} U_{x \mu}^{\dagger} \phi_{x}\right) \sim-\kappa \sum \phi^{\dagger} \square(U) \phi,
$$

which is the usual kinetic term for the scalar field.

Similarly, the HD gauge-fixing term (2.3) becomes, in the Higgs picture,

$$
S_{\mathrm{GS}}^{\phi}=\tilde{\kappa}\left(\sum \phi^{\dagger} \square^{2}(U) \phi-\sum \mathscr{B}^{2}\right),
$$

where,

$$
\mathscr{B}_{x}=\sum_{\mu}\left(\overline{\mathscr{A}}_{x-\mu, \mu}+\overline{\mathscr{A}}_{x \mu}\right)^{2} / 4, \text { with } \overline{\mathscr{A}}_{x \mu}=\operatorname{Im}\left(\phi_{x}^{\dagger} U_{x \mu} \phi_{x+\mu}\right)
$$

The total action, in the Higgs picture, thus assumes the form:

$$
S^{\phi}=S_{\mathrm{W}}+S_{\mathrm{GS}}^{\phi}+S_{\mathrm{ct}}^{\phi}
$$

where the standard Wilson term $S_{\mathrm{W}}$ is gauge invariant and hence does not pick up the lgdof when the functional integral integrates along the gauge orbit. 
The gauge invariance as found in the standard Wilson term $S_{\mathrm{W}}$ alone is the target symmetry under the gauge transformations:

$$
U_{x \mu} \rightarrow \mathrm{g}_{x} U_{x \mu} \mathrm{g}_{x+\mu}^{\dagger}, \quad \mathrm{g}_{x} \in U(1)
$$

However, the total action (2.12) in the Higgs picture has an enlarged (unphysical) symmetry under the transformations

$$
U_{x \mu} \rightarrow \mathrm{h}_{x} U_{x \mu} \mathrm{h}_{x+\mu}^{\dagger}, \quad \phi_{x} \rightarrow \mathrm{h}_{x} \phi_{x}, \quad \mathrm{~h}_{x} \in U(1) .
$$

We would call the local symmetries given by (2.13) and (2.14) respectively as the g-symmetry (target physical symmetry) and the h-symmetry.

Putting $\phi_{x}=1$ in the expression for the action $S^{\phi}$ in the Higgs picture (2.12) recovers the action (2.1), called the action in the vector picture. Given the Haar measure of the functional integrals, theories given by the two actions (2.1) and (2.12) are completely equivalent.

With vanishing $\tilde{\kappa}$, the theory approaches an Abelian gauge-Higgs system.

With zero gauge coupling $g=0$, we have $U_{x \mu}=1$ for all the links of the lattice. This is known as the reduced limit. The action of the reduced model is given by,

$$
S[\phi]=-\kappa \sum_{x} \phi_{x}^{\dagger}(\square \phi)_{x}+\tilde{\kappa} \sum_{x}\left\{\phi_{x}^{\dagger}\left(\square^{2} \phi\right)_{x}-b_{x}^{2}\right\},
$$

where $b_{x}$ is the appropriate modification of $\mathscr{B}_{x}$ of Eq. (2.11) with $U_{x \mu}=1$.

The reduced model action is invariant under the global transformations

$$
\phi_{x} \rightarrow \mathrm{h} \phi_{x}
$$

where $\mathrm{h} \in U(1)_{\text {global }}$ is independent of the lattice site.

Following [5], we can gain useful insight into the phase diagram in the region of small $g$ and large $\tilde{\kappa}$ by doing a simple-minded calculation. We start from the action (2.1) in the so-called vector picture, and use the property that the action has an absolute minimum at $U_{x \mu}=\exp \left(\operatorname{iag} A_{\mu}(x)\right)=1$. Near this point, the action can be expanded in powers $g$ in the constant field approximation, i.e., by neglecting derivatives of the gauge field. This leads to an expression for a classical potential density in powers of the gauge coupling $g$ :

$$
V_{\mathrm{cl}}\left(A_{\mu}\right)=\kappa\left(g^{2} \sum_{\mu} A_{\mu}^{2}+\ldots\right)+\frac{g^{6}}{2} \tilde{\kappa}\left\{\left(\sum_{\mu} A_{\mu}^{2}\right)\left(\sum_{\mu} A_{\mu}^{4}\right)+\ldots\right\},
$$

where terms with higher powers of $g^{2}$ are indicated by the ellipses. The classical potential density is expected to be a reasonable approximation at small $g$. However, as it turns out from numerical simulations, the classical potential density (2.17) produces a good qualitative picture of the new universality class in regions of the parameter space where the gauge coupling $g$ is not very small and $\tilde{\kappa}$ is only sufficiently large, depending on the value of $g$.

Inspection of the expression for $V_{\mathrm{cl}}$ (2.17) immediately leads to a critical surface defined by

$$
\kappa \equiv \kappa_{\mathrm{FM}-\mathrm{FMD}}(g, \tilde{\kappa})=0,
$$


where the gauge boson (photon) is rendered massless.

Minimization of $V_{\mathrm{cl}}$ (2.17) with respect to $g A_{\mu}$ shows that the classical potential density has two different minima at $g A_{\mu}=0$ for $\kappa \geq \kappa_{\mathrm{FM}-\mathrm{FMD}}$, and at $g A_{\mu}= \pm\left(\frac{\left|\kappa-\kappa_{\mathrm{FM}-\mathrm{FMD}}\right|}{6 \tilde{\kappa}}\right)^{1 / 4}$ for $\kappa<\kappa_{\mathrm{FM}-\mathrm{FMD}}$. Hence, in the quantum theory at small $g$ and large $\tilde{\kappa}$, it is expected that tuning $\kappa$ to $\kappa_{\mathrm{FM}-\mathrm{FMD}}(g, \tilde{\kappa})$ signals a new continuous phase transition, within the broken phase, with a vector condensate $\left\langle g A_{\mu}\right\rangle$ as an order parameter. The phase with the vector condensate is the novel phase and is called Ferromagnetic Directional (FMD) phase across all versions of the theory, including the theory in the reduced limit. Obviously the FMD phase breaks the rotational symmetry, and no Lorentz covariant continuum limit is obtainable from within the FMD phase. Hence, continuum limit is to be taken by approaching the continuous FM-FMD transition from the so-called Ferromagnetic (FM) phase.

Earlier investigations done in $[5,9]$ at weak gauge couplings are consistent with the above picture.

For weak couplings, these studies confirmed a phase diagram with generic features as given in the left panel of Fig. 1 at gauge coupling $g=1$, approximately the largest gauge coupling exhibiting all the features of the phase diagram at weak gauge couplings $(g<1)$. The nomenclature of the phases in this theory has been taken as per the phases in the so-called reduced model [9]. The regular broken phase, FM (with ferromagnetic order) is characterized by a massive photon and a massive scalar, the PM (for paramagnetic) phase is the disordered (symmetric) phase having massless photons, and finally the new FMD (ferromagnetic-directional) phase is the spatially modulated ordered phase that breaks Euclidean rotational symmetry (there is also an anti-ferromagnetic or AM phase with staggered order, not to be discussed further in this study). Photon and scalar masses scale by approaching the continuous FM-PM transition from the FM phase, leading to a continuum gauge-Higgs theory. A sufficiently large $\tilde{\kappa}$ (and small $g$ ) ensures a satisfactory continuum limit with only the photon mass scaling (thereby recovering gauge symmetry and decoupling the scalars) at the FM-FMD phase transition by tuning a single parameter $\kappa$ from the FM side.
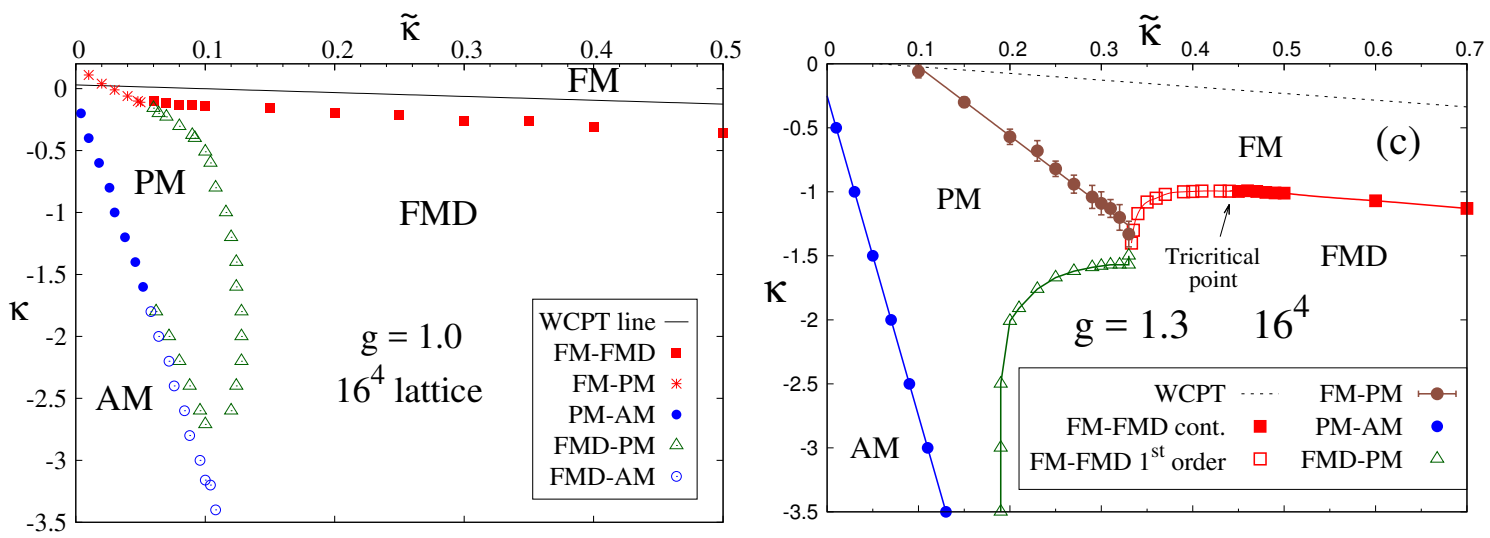

Figure 1: Phase diagram in the $(\tilde{\kappa}, \kappa)$ plane at gauge coupling $g=1.0$ on the left and at $g=1.3$ on the right, on $16^{4}$ lattice

\subsection{Results at strong gauge coupling}

Vacuum expectation values of quantities that were measured on equilibrated gauge field con- 
figurations on $L^{4}$ (or $L^{3} T, L<T$ for propagators) lattices, are the average plaquette $E_{\mathrm{P}}$, the gauge field mass term $E_{\kappa}=\frac{1}{4 L^{4}}\left\langle\sum_{x, \mu} \operatorname{Re} U_{x \mu}\right\rangle$, and the lattice version $V$ of the vector condensate $\left\langle A_{\mu}\right\rangle$. The vector condensate $V$ is the order parameter for the FM-FMD transition. It is zero for all other phases except FMD. For first order FM-FMD transition, the quantity $E_{\kappa}$ goes through a finite jump when plotted against $\kappa$. However, for continuous FM-FMD transition, the finite jump vanishes and $E_{\kappa}$ is continuous across the transition. Out of all the phase transitions to be presented here, the one of prime importance to us is the FM-FMD, and the location and nature of this transition including the tricritical points naturally attracted most of our attention.

In Fig. 1 all data points represented by solid (filled) symbols signify a continuous phase transition, while all data points represented by empty (unfilled) symbols signify a first order transition. Accordingly, one would find that FM-PM and PM-AM phase transitions are continuous and PMFMD phase transition is first order, for all gauge couplings investigated.

For values of the gauge coupling $g>1$, the FM-FMD phase transition develops a first order part for smaller values of $\tilde{\kappa}$, as seen for $g=1.3$ in the left panel of Fig. 1. At $g=1.1$ (not shown), in our simulations on $16^{4}$ lattice, the FM-FMD phase transition first shows a little glimpse of its first order part for small values of $\tilde{\kappa}$ and then quickly turns itself into a continuous transition at a tricritical point at $(\tilde{\kappa}, \kappa) \sim(0.14,-0.33)$ and remains continuous for larger values of $\tilde{\kappa}$. As the gauge coupling $g$ is further increased, the location of the tricritical point in the $(\tilde{\kappa}, \kappa)$-plane shifts to larger $\tilde{\kappa}$ and more negative $\kappa$. In other words, the first order part of the FM-FMD phase transition extends quite rapidly with increase of the gauge coupling. However, it appears from our numerical simulations (which includes gauge couplings $g>1.5$, corresponding data not shown here) that, given a large gauge coupling there is always a sufficiently large $\tilde{\kappa}$ beyond which the FM-FMD transition is continuous. In addition, the FM-FMD transition overall shifts to larger negative $\kappa$ values at stronger gauge couplings.

While the bare WCPT done around the point $g=0$ and $\tilde{\kappa}=\infty$ has limited range of applicability, there exists no phase transitions between the WCPT corner of the 3-dimensional coupling parameter space (viz., $g=0$ and $\tilde{\kappa}=\infty$ ) and any point on the continuous part of the FM-FMD transition at a strong gauge coupling and a large enough $\tilde{\kappa}$. The schematic phase diagram in the 3 -dimensional parameter space $(g, \tilde{\kappa}, \kappa)$ is displayed on the left panel in Fig. 2. Kindly note that $\kappa=0$ surface is located slightly below the top surface of the 3 -dimensional box presented in the figure. The diagram is drawn based on available data on phase transitions and interpolations and extrapolations. The continuity of the entire FM-FMD transition surface (bounded by the tricritical line starting at $g=1.1$ ) up to the WCPT corner is clearly evident when we look at the 3-dimensional phase diagram. Hence it is natural to expect that this whole region falls under the same universality class and the continuum physics obtainable should be no different from that near the weak gauge coupling region.

The inverse of photon propagator (2-point correlator of $\operatorname{Im} U_{x \mu}$ ) in momentum space was also measured and is plotted against the square of lattice momentum $\hat{p}^{2}$ (discrete on a finite box) on the right panel in Fig. 2 for the continuous part of the FM-FMD transition, staying in the FM phase. Inset shows a gradually vanishing photon mass $\left(y\right.$-intercept), as $\kappa$ approaches $\kappa_{\mathrm{FM}-\mathrm{FMD}}(\sim-1.07)$ for the given fixed $\tilde{\kappa}(0.6)$, suggesting an expected scaling of the photon mass at the transition and recovery of gauge symmetry. The slope of the fitted straight lines, in the main figure, suggests a field renormalization constant $Z$ that is not unity. However, the figure shows that the slope increases 

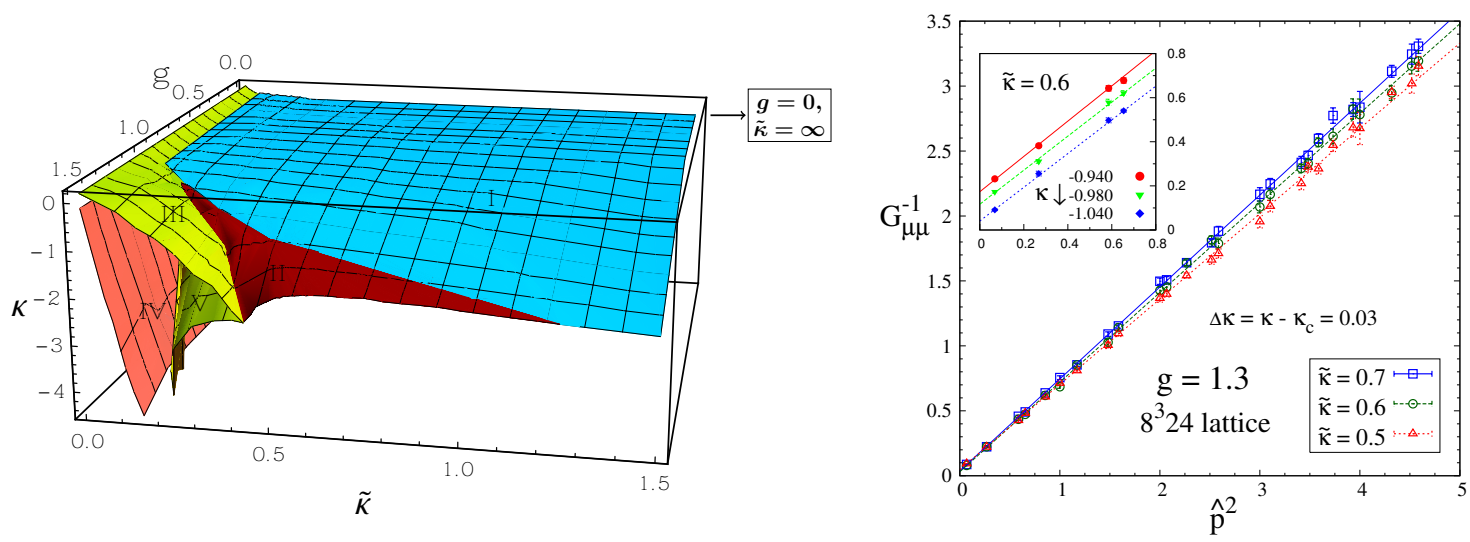

Figure 2: Left: Schematic phase diagram in the 3-dimensional $(g, \tilde{\kappa}, \kappa)$ parameter space at gauge coupling based on available data on $16^{4}$ lattice. Surfaces of different phase transitions are labelled by: I: FM-FMD (continuous), II: FM-FMD (first order), III: FM-PM (continuous), IV: PM-AM (continuous), V: PM-FMD (first order). A tricritical line separating the first order and the continuous FM-FMD transitions emerges at $g>1$ and continues to move towards larger $\tilde{\kappa}$ at stronger gauge coupling $g$. The arrow at the top right points to the WCPT corner $(g=0, \tilde{\kappa} \rightarrow \infty)$. Right: Inverse photon propagators in the FM phase near FM-FMD transition at $g=1.3$ and at three values of $\tilde{\kappa}$ for which the transition is continuous. Inset shows scaling of the photon mass as FM-FMD transition is approached from FM side.

with increasing $\tilde{\kappa}$. It seems reasonable to expect the slope to approach unity at large $\tilde{\kappa}$, consistent with WCPT at $g=0$ or $\tilde{\kappa}=\infty$.

\section{Equivariant BRST for $S U(2)$ lattice gauge theory}

\subsection{Introduction}

Equivariant BRST (eBRST) formalism on the lattice was suggested by Schaden [10] for $S U(2)$ and later extended by Golterman and Shamir [11] for $S U(N)$ gauge theories. In this formalism, the gauge-fixing is done on a coset leaving minimally a Cartan subgroup. The nilpotency of usual BRST is modified in eBRST in the following sense: a double variation results in an infinitesimal gauge variation of the subgroup. Hence nilpotency is still valid for all operators invariant under the subgroup. Another important consequence, not seen in usual BRST, is the presence of a 4-ghost term, which is responsible for evading the no-go theorem. Golterman and Shamir was able to write down an extended eBRST action invariant under eBRST and anti-eBRST. It was also possible to write down a lattice action for the extended eBRST theory keeping all the internal symmetries.

We have initiated a first-time numerical investigation of the eBRST theory for the gauge group $S U(2)$ [4]. In this special case of $S U(2)$, the extended eBRST formalism reduces to the ordinary eBRST, and the only possibility of the coset is $S U(2) / U(1)$. The ghost and anti-ghost fields $C$ and $\bar{C}$, and the usual auxiliary fields $B$ reside only in the coset.

For the $S U$ (2) Yang-Mills theory, the gauge field is split as in the following,

$$
V_{\mu}^{a} \tau_{a}=A_{\mu}^{i} \tau_{i}+W_{\mu}^{\alpha} \tau_{\alpha}, \quad \text { with } a=1,2,3, i=3 \& \alpha=1,2
$$


The generators $\tau_{a}=\sigma_{a} / 2$, where $\sigma_{a}$ are the Pauli matrices. The ghost sector fields are a linear combination of the generators $\tau_{1}$ and $\tau_{2}$. With the auxiliary field $B$ integrated out, the total Lagrangian density for the gauge-fixing part is

$$
\begin{aligned}
\mathscr{L}_{G F}^{S U(2)} & =\frac{1}{\tilde{g}^{2}} \operatorname{tr}\left(D_{\mu}(A) W_{\mu}\right)^{2}+\mathscr{L}_{g h}^{(2)}+\mathscr{L}_{g h}^{(4)}, \text { where, } \\
\mathscr{L}_{g h}^{(2)} & =-2 \operatorname{tr}\left(\bar{C} D_{\mu}(A) D_{\mu}(A) C\right)+2 \operatorname{tr}\left(\left[W_{\mu}, \bar{C}\right]\left[W_{\mu}, C\right]\right), \\
\mathscr{L}_{g h}^{(4)} & =-\operatorname{tr}(\widetilde{X})^{2}, \text { with } \widetilde{X}=i\{C, \bar{C}\} .
\end{aligned}
$$

$\mathscr{L}_{g h}^{(2)}$ and $\mathscr{L}_{g h}^{(4)}$ are respectively the two and four ghost terms and $\tilde{g}^{2}=\xi g^{2}$ is the gauge-fixing coupling. The gauge-fixing function $D_{\mu}(A) W_{\mu}=\partial_{\mu} W_{\mu}+i\left[A_{\mu}, W_{\mu}\right]$ is in the coset $S U(2) / U(1)$ and is covariant under $U(1)$ gauge transformations.

\subsection{Lattice action, invariance theorem and the reduced model}

The total eBRST gauge-fixed $S U(2)$ action on the lattice is given as

$$
S=S_{W}+S_{g f},
$$

where $S_{W}$ is the usual Wilson plaquette action,

$$
S_{W}=\frac{\beta}{2} \sum_{x, \mu<v} \operatorname{Re} \operatorname{tr}\left[1-P_{x \mu v}\right], \quad \beta=4 / g^{2} .
$$

The eBRST gauge-fixing term, with introduction of a new single-component real auxiliary field $\rho_{x}=\rho_{x 3} \tau_{3}$ to take care of the four-ghost interaction term, is given as

$$
\begin{aligned}
S_{g f} & =\frac{1}{2 \tilde{g}^{2}} \sum_{x \alpha}\left(D_{\mu}^{-} \mathscr{W}_{x \mu}\right)_{\alpha}^{2}+\frac{1}{2 \tilde{g}^{2}} \sum_{x}\left(\rho_{x 3}\right)^{2}+\sum_{x y \alpha \beta} \bar{C}_{x \alpha} M_{x \alpha, y \beta} C_{y \beta} \\
& =\tilde{\kappa} \sum_{x \alpha}\left(D_{\mu}^{-} \mathscr{W}_{x \mu}\right)_{\alpha}^{2}+\tilde{\kappa} \sum_{x}\left(\rho_{x 3}\right)^{2}+\sum_{x y \alpha \beta} \bar{C}_{x \alpha} M_{x \alpha, y \beta} C_{y \beta} .
\end{aligned}
$$

The lattice eBRST gauge-fixing function,

$$
D_{\mu}^{-} \mathscr{W}_{x \mu}=\mathscr{W}_{x \mu}-U_{x-\mu, \mu}^{\dagger} \mathscr{W}_{x \mu} U_{x-\mu, \mu},
$$

with the lattice equivalent of the coset gauge field $W_{\mu}(x)$,

$$
\mathscr{W}_{x \mu} \equiv-i\left[U_{x \mu} T_{i} U_{x \mu}^{\dagger}, T_{i}\right]=W_{x \mu}+O\left(V^{2}\right),
$$

goes to $D_{\mu}(A) W_{\mu}$ in the classical continuum limit.

The ghost matrix, $M(U, \rho)=\Omega(U)+R(\rho)$, is real, with the gauge-field dependent $\Omega$ being symmetric and $R_{x \alpha, y \beta}=\delta_{x y} \rho_{x 3} f_{3 \alpha \beta}$ being antisymmetric in the coset indices. The matrix element $\Omega_{x \alpha, y \beta}$ is explicitly given by

$$
\begin{gathered}
\Omega_{x \alpha, y \beta}=2 \delta_{\alpha \beta} \delta_{x y} \operatorname{tr}\left(U_{y-\mu, \mu}^{\dagger} \tau_{3} U_{y-\mu, \mu} \tau_{3}+U_{y \mu} \tau_{3} U_{y \mu}^{\dagger} \tau_{3}\right) \\
-2 \varepsilon_{\alpha \delta} \varepsilon_{\beta \gamma} \delta_{x, y-\mu} \operatorname{tr}\left(U_{y \mu} \tau_{\gamma} U_{y \mu}^{\dagger} \tau_{\delta}\right) \\
-2 \varepsilon_{\alpha \gamma} \varepsilon_{\beta \delta} \delta_{x, y+\mu} \operatorname{tr}\left(U_{y \mu} \tau_{\gamma} U_{y \mu}^{\dagger} \tau_{\delta}\right) .
\end{gathered}
$$


In Eq.3.5, the parameter $\tilde{\kappa} \equiv 1 / 2 \tilde{g}^{2}=1 / 2 \xi g^{2}$ has been introduced as in Eq.2.7. The right hand side of Eq.3.5 is the starting point of the numerical calculation. The simulation is done in the parameter space of $\beta$ and $\tilde{\kappa}$. This will be denoted as the full theory from now onwards, as opposed to the reduced model to be discussed later.

A mass term for the lattice coset gauge field $\mathscr{W}_{x \mu}$ and for the ghost field $C_{x}$, together invariant under on-shell eBRST transformations, can be added in the action in Eq. 3.3, as

$$
S_{\text {mass }}=m^{2} \sum_{x}\left[-4 \tilde{\kappa} \operatorname{tr}\left(U_{x \mu} \tau_{3} U_{x \mu}^{\dagger} \tau_{3}\right)+2 \operatorname{tr}\left(\bar{C}_{x} C_{x}\right)\right] .
$$

However, the mass term, although eBRST-invariant, is not eBRST-exact, i.e., it cannot be expressed as an eBRST variation of something else.

It can be explicitly shown that the no-go theorem is evaded in the eBRST formalism since the eBRST partition function can be shown to be a non-zero constant. This turns out to be true also with the addition of the mass term (3.9). Unlike in the BRST case, the ghost integral is non-zero due to the presence of the four-ghost term in the eBRST formalism. A direct consequence of the above is an invariance theorem that makes the expectation value of a gauge-invariant operator in the eBRST theory equal to that in the unfixed theory. In the massive eBRST case, however, the equality should hold for unfixed theory with massive gauge fields in the coset. The invariance theorem is a rigorous result from eBRST symmetry on a finite lattice.

The theory on the gauge orbit is the so-called reduced model. It is an interacting theory of the longitudinal gauge degrees of freedom (lgdof) and ghost fields. The reduced limit is taken by setting the gauge coupling $g=0$. As a consequence of the invariance theorem, the reduced model is found to be topological field theory (TFT), as the partition function of the reduced model is just a number independent of the gauge-fixing coupling $\tilde{g}$.

As described in the context of the $U(1)$ theory previously, the full theory action $S[U]$ is given a gauge transformation to obtain $S\left[\phi U \phi^{\dagger}\right]$ with $\operatorname{lgdof} \phi$. The gauge fields $U$ are then set to 1 to arrive at the reduced model action $S^{\text {red }}$. The action of the reduced model of the eBRST gauge-fixed $S U(2)$ theory, with lgdof as group-valued $\phi$ fields, is given as follows:

$$
\begin{aligned}
S^{\mathrm{red}}=S_{g f}^{\mathrm{red}} & =2 \tilde{\kappa} \sum_{x} \operatorname{tr}\left[-2\left(\phi_{x} \phi_{x+\mu}^{\dagger} \tau_{3} \phi_{x+\mu} \phi_{x}^{\dagger} \tau_{3}+\phi_{x} \phi_{x-\mu}^{\dagger} \tau_{3} \phi_{x-\mu} \phi_{x}^{\dagger} \tau_{3}\right) \times(v-\text { term })\right. \\
& \left.+\frac{1}{2}\left(\phi_{x} \phi_{x+\mu}^{\dagger} \tau_{3} \phi_{x+\mu} \phi_{x}^{\dagger}+\phi_{x} \phi_{x-\mu}^{\dagger} \tau_{3} \phi_{x-\mu} \phi_{x}^{\dagger}\right) \times(v-\text { term })\right] \\
& +\tilde{\kappa} \sum_{x}\left(\rho_{x 3}\right)^{2}+\sum_{x y \alpha \beta} \bar{C}_{x \alpha} M_{x \alpha, y \beta}^{\mathrm{red}} C_{y \beta},
\end{aligned}
$$

where $M_{x \alpha, y \beta}^{\text {red }}$ is the reduced-model-ghost matrix.

The eBRST-invariant mass term of the reduced model,

$$
S_{\text {mass }}^{\mathrm{red}}=m^{2} \sum_{x}\left[-4 \tilde{\kappa} \operatorname{tr}\left(\phi_{x} \phi_{x+\mu}^{\dagger} \tau_{3} \phi_{x+\mu} \phi_{x}^{\dagger} \tau_{3}\right)+2 \operatorname{tr}\left(\bar{C}_{x} C_{x}\right)\right] .
$$

can also be added to the action in Eq. 3.10.

In addition to its eBRST-invariance, the reduced model is invariant under the remnant local $U(1)$ symmetry, corresponding to the unfixed part of the theory. The local $U(1)$ transformation in the reduced model acts on the $\phi$ field from the left, hence denoted here as $U(1)_{L}$ :

$$
\phi_{x}^{\prime}=\mathrm{h}_{x} \phi_{x}, \quad \mathrm{~h}_{x}=\exp \left(\mathrm{i} \theta_{x} \tau_{3}\right) \in U(1)_{L} .
$$


The action is also invariant under a global $S U(2)_{R}$ transformation, acting on the $\phi$ fields from the right, since all the terms have the $\phi$ fields present as $\phi \phi^{\dagger}$ combination:

$$
\phi_{x}^{\prime}=\phi_{x} \mathrm{~g}, \quad \mathrm{~g} \in S U(2)_{R} .
$$

The ghost fields are Grassmann-valued and are not directly implemented in the computer. However, they can be replaced by real-valued fields, similar to what is done in the pseudofermion method used for fermions. The integration of the ghost fields in the partition function leads to,

$$
\int \mathscr{D C} \mathscr{D} \bar{C} \exp (-\bar{C} M C)=\operatorname{det} M=|\operatorname{det} M| \operatorname{sign}(\operatorname{det} M) .
$$

$|\operatorname{det} M|$ can be simulated using HMC by introducing a real-valued scalar ("pseudo-ghost") field $\varphi$,

$$
|\operatorname{det} M|=\sqrt{\operatorname{det}\left(M M^{T}\right)}=\int \mathscr{D} \varphi \exp \left(-(1 / 2) \varphi^{T}\left(M M^{T}\right)^{-1} \varphi\right) .
$$

Note that the notation $\varphi$ is different from the $\phi$ field in the reduced model.

As a first calculation, one can ignore the sign of the ghost matrix determinant, which can be shown to be real, and simulate the full theory and the reduced model using HMC without tracking the sign. In the full theory, by computationally verifying the validity of the invariance theorem, assuming unbroken eBRST, one can then identify regions of parameter space in $(\beta, \tilde{\kappa})$ where sign changes occur and where they do not. A signature within the HMC that identifies increasingly poor conditioning of the ghost matrix usually accompanied by a few eigenvalues getting small is the divergence of the number of iterations for ghost matrix inversion and the related force term during the course of the MD trajectory of the HMC. Such situations usually lead to the failure of HMC.

We have studied the full theory and the reduced model using HMC, ignoring the sign. We have also done, in order to track the sign of the ghost matrix determinant, a first-time stochastic implementation of the tunneling HMC (sTHMC) [12] in the full theory. However, in this write-up, we are not going to present any results from STHMC, since we are still to achieve a good control over the efficiency of sTHMC.

\subsection{Results for the eBRST gauge theory and the reduced model}

It has been shown by analysis of perturbative 1-loop renormalization group (RG) equations in [13] that the coupling $\tilde{g}$ in the gauge fixing sector $\left(\tilde{g}^{2}=\xi g^{2}=1 /(2 \tilde{\kappa})\right)$, where $\xi$ is the gauge fixing parameter) is also asymptotically free, just like the non-Abelian gauge coupling $g$. Hence, dimensional transmutation takes place and both these couplings can effectively become strong at infrared scales $\Lambda$ and $\tilde{\Lambda}$ corresponding to $g$ and $\tilde{g}$. According to the findings in [13], two possibilities exist: one where the $\Lambda$ parameters are of the same order, the other where the $\tilde{\Lambda} \gg \Lambda$. Obviously, it is an interesting question to ask about the nature of the strong coupling theory on the orbit, i.e., the reduced model. Can strong dynamics of the lgdof lead to a SSB?

It was demonstrated in Ref. [14] through a toy model, that a phase transition can indeed occur in a TFT through a non-trivial effective potential. The authors provided evidence of the existence of a broken phase in the reduced model through a combination of strong coupling and mean-field techniques: the global $S U(2)_{R}$ symmetry of the action spontaneously breaks in the manner, $S U(2)_{R} \rightarrow U(1)_{R}$. Although the existence of the SSB can only be verified through a 
controlled non-perturbative calculation, this and its effect on the full theory raises very interesting possibilities. Our numerical simulations are aimed at finding the truth about these possibilities.

The quantity $\tilde{A}_{x}[14]$ can be used to signal the symmetry breaking of the global $S U(2)_{R} \rightarrow$ $U(1)_{R}$ in the reduced model. The expectation value

$$
\left\langle\tilde{A}_{x}\right\rangle=\left\langle\phi_{x}^{\dagger} \tau_{3} \phi_{x}\right\rangle
$$

transforms in the adjoint representation of $S U(2)_{R}$ and is invariant under the local $U(1)_{L}$ transformation. It is to be noted that, $\tilde{A}_{x}$ is not invariant under the modified eBRST transformation, but since it is not a total eBRST variation, it cannot signal a possible spontaneous breaking of eBRST symmetry.

In the numerical simulation, we have studied the length of the average $\tilde{A}$ vector averaged over many ensembles, given for a lattice volume $V$ by

$$
\langle|\tilde{A}|\rangle=\left\langle\sqrt{\sum_{i=1}^{3}\left(\frac{1}{V} \sum_{x} \pi_{x i}\right)^{2}}\right\rangle, \quad \text { where } \pi_{x i}=\frac{1}{2} \operatorname{tr}\left(\tilde{A}_{x} \tau_{i}\right) .
$$

$\langle|\tilde{A}|\rangle \neq 0$ would signal the SSB: $S U(2)_{R} \rightarrow U(1)_{R}$ in the reduced model.

In our numerical study, a symmetry breaking seed

$$
S_{\text {seed }}=-h \operatorname{tr}\left(\tau_{3} \tilde{A}_{x}\right),
$$

is also used. If a seed is used, it is sufficient to compute the expectation value of $\pi_{3}$, the $\tau_{3}$ component of the average $\tilde{A}$ for each configuration, and then gradually go to the limit $h \rightarrow 0$ for the order parameter for the SSB. We have checked that, within the statistical errors, both procedures produce the same result for the order parameter.

Since the gauge-fixing coupling, $\tilde{g}$ (related to $\tilde{\kappa}$ as $\tilde{\kappa}=1 /\left(2 \tilde{g}^{2}\right)$ ), is asymptotically free, the HMC algorithm faces critical slowing down as we make $\tilde{g}$ weaker, or equivalently $\tilde{\kappa}$ larger. On the other hand, in the region where the gauge-fixing coupling $\tilde{g}$ is strong or $\tilde{\kappa}$ small $(<1.0)$, we face problems with ghost matrix inversion using our BiCGStab inverter used for the purpose. For the range of $\tilde{\kappa}$ between 1.0 and 10.0, the HMC on $8^{4}$ lattices runs reasonably well for the reduced model (with no mass terms added), although to get acceptance rates around $50 \%$, we needed to keep the MD step-size $\sim 0.005$ and the number of MD steps in a trajectory within 10 . This already shows that the simulation in these systems is very difficult. Fortunately, despite the small step-size and the number of MD steps, the integrated autocorrelation time is within acceptable limits $(<200)$ and the system shows the desired fluctuation of the observables in its field configurations. As we increase the lattice size to $12^{4}$ and $16^{4}$ (data not presented in this write-up), the autocorrelation times increase quite rapidly, however, still within manageable limits of our resources for $12^{4}$.

The left panel in Fig. 3 clearly indicates presence of SSB in the reduced model for a wide region of $\tilde{\kappa}$. There appears to be a sharp transition to symmetry restoration at a small $\tilde{\kappa}$ very near zero, roughly consistent with mean field calculations [14]. The broken phase appears to continue to exist all the way to arbitrarily large $\tilde{\kappa}$, i.e., the perturbative region of $\tilde{g}$.

It is of course very interesting to think about the effect of the SSB in the reduced model on the full theory when the transverse gauge degrees of freedom are turned back on. However, the 

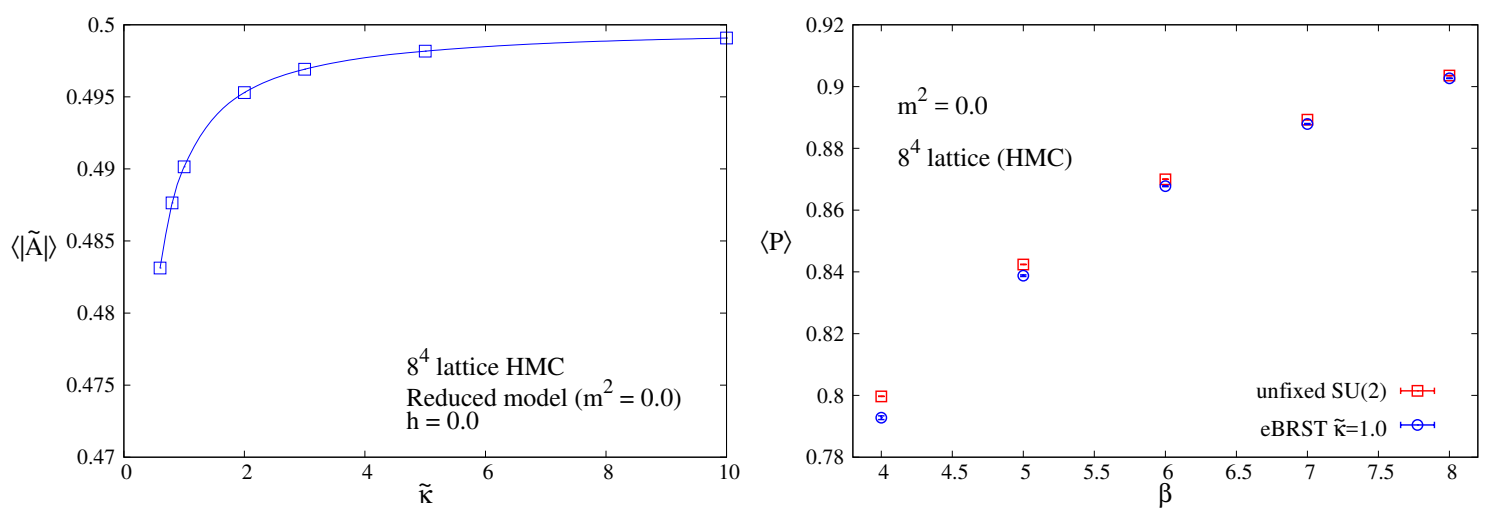

Figure 3: Left: Variation of $\langle|\tilde{A}|\rangle$ with $\tilde{\kappa}$ on $8^{4}$ lattices, with $m^{2}=0.0$ and $h=1.0$. Right: Comparison of plaquette expectation values $\langle P\rangle$ of unfixed and eBRST gauge-fixed theories at different $\beta$ for a fixed $\tilde{\kappa}=1.0$.

numerical simulation in the full theory is even harder. We have comprehensively scanned the region $\beta=3.0-8.0$ and $\tilde{\kappa}=0.1-10$ for $m^{2}=0.0$ with the HMC algorithm. Unlike in the reduced model, there is no region found in the full theory in the scanned $\beta-\tilde{\kappa}$ parameter space where the ghost matrix inversions take place without issues. Generally, the number of iterations of the BiCGStab inverter blow up or the inversion fails at some point during the field updation. The severity of the inversion problem increases with decreasing $\beta$ and/or decreasing $\tilde{\kappa}$, i.e., with stronger gauge and gauge-fixing couplings $g$ and $\tilde{g}$ respectively. The symptoms generally indicate the presence of near-zero eigenvalues of the ghost matrix. On the other hand, critical slowing down, in the form of decreasing acceptance rate, is also found with increasing $\beta$ and/or $\tilde{\kappa}$, as also seen in the reduced model for large $\tilde{\kappa}$.

We have calculated the expectation value of the gauge-invariant plaquette for both unfixed and gauge-fixed theories on the lattice to study the validity of the invariance theorem. Without a symmetry breaking seed, the eBRST symmetry is exact, and on our finite lattices the invariance theorem is strictly applicable. Hence under these conditions, any violation of the invariance theorem appears to be an indicator of sign changes of the ghost determinant, something that our HMC algorithm ignores.

The right panel in Fig. 3 shows that the expectation value of the plaquette computed in the eBRST theory becomes increasingly closer to the value computed in the unfixed theory with increase of $\beta$. At $\beta=8.0$, the largest shown in the figure, the values are almost equal to each other. This observation coupled with the trend of the inversion problems with $\beta$ is consistent with the expectation that, in the perturbative limit $(\beta \rightarrow \infty)$ the ghost matrix determinant does not show any change of sign. It seems reasonable to conclude that there are more and more sign changes as $\beta$ decreases.

Approximate manifestation of the invariance theorem, at least at our larger $\beta$ values, is also a validation for the eBRST gauge fixed theory as an alternate formulation of non-Abelian gauge theory on the lattice. From the invariance theorem it also follows that the expectation value of a gauge invariant operator should be independent of the gauge-fixing coupling. This was also verified for the plaquette (not shown here). 


\section{Conclusion and future directions}

We have given an account of our study of non-perturbatively gauge-fixed lattice gauge theory, with chiral gauge theory as a motivation and also as an alternate formulation of lattice gauge theories. The HD gauge-fixing proposal for the compact $U(1)$ lattice gauge theory was shown to work also at strong gauge couplings $(g>1)$ in the same way it was shown earlier to work at weak gauge couplings. A triciritical line, obtained at strong gauge couplings in the 3-dimensional phase diagram, appears to be the only candidate for any possible non-trivial behaviour. Through verification of the invariance theorem, we have also numerically validated the eBRST gauge fixing formalism for the SU(2) lattice gauge theory. Strong dynamics of the lgdof results in a SSB in the theory on the gauge orbit, i.e., the reduced model, which is a TFT. It is interesting to ask what would be the consequence of the strong dynamics of the lgdof in the full theory, that includes the transverse gauge degrees of freedom. From strong coupling and mean field analysis [14], there is a phase with no mass gap. However, to investigate these issues, we need to have better algorithms and a way to track the sign changes of the ghost matrix determinant. Efforts are underway.

Acknowledgement: The authors are indebted to M. Golterman and Y. Shamir for numerous discussions. The computing facilities at the Theory Division, SINP provided by fundings from DAE, Govt. of India is greatly acknowledged.

\section{References}

[1] H. Neuberger, Phys. Lett. B 183 (1987) 337.

[2] A. K. De and M. Sarkar, Phys. Rev. D 93 (2016) no.11, 114504.

[3] A. K. De and M. Sarkar, JHEP 1710 (2017) 125.

[4] A. K. De and M. Sarkar, manuscript in preparation.

[5] M. F. L. Golterman and Y. Shamir, Phys. Lett. B 399 (1997) 148.

[6] W. Bock, M. F. L. Golterman and Y. Shamir, Phys. Rev. Lett. 80 (1998) 3444.

[7] S. Basak and A. K. De, Phys. Rev. D 64 (2001) 014504.

[8] W. Bock, K. C. Leung, M. F. L. Golterman and Y. Shamir, Phys. Rev. D 62 (2000) 034507.

[9] W. Bock, M. F. L. Golterman and Y. Shamir, Phys. Rev. D 58 (1998) 054506.

[10] M. Schaden, Phys. Rev. D 59 (1999) 014508.

[11] M. Golterman and Y. Shamir, Phys. Rev. D 70 (2004) 094506.

[12] M. Golterman and Y. Shamir, Phys. Rev. D 76 (2007) 094512.

[13] M. Golterman and Y. Shamir, Phys. Rev. D 73 (2006) 014510.

[14] M. Golterman and Y. Shamir, Phys. Rev. D 87 (2013) no.5, 054501. 